Pure and Applied Mathematics Quarterly

Volume 1, Number 1, 165-179, 2005

\title{
The integral K-theoretic Novikov conjecture for geometrically finite groups
}

\author{
Lizhen Ji
}

\begin{abstract}
In this note, we prove the integral K-theoretic Novikov conjecture for geometrically finite discrete subgroups of semisimple Lie groups of rank 1 , in particular geometrically finite Kleinian groups.
\end{abstract}

\section{Introduction}

An important conjecture in topology is the Novikov conjecture on (oriented) homotopy invariance of the higher signatures, which can be formulated equivalently as the rational injectivity of the assembly map in algebraic surgery theory, i.e., L-theory (see $[\mathrm{FRR}])$. The injectivity of the assembly map is called the integral Novikov conjecture in L-theory. There are also assembly maps in algebraic Ktheory and the theory of $C^{*}$-algebras, and the rational injectivity of each assembly map is called the Novikov conjecture, and the injectivity of the assembly map the integral Novikov conjecture in the corresponding theory.

For torsion free discrete subgroups of Lie groups, the integral Novikov conjecture in $C^{*}$-algebras was proved by Kasparov in [Ka]. For torsion free discrete subgroups of linear Lie groups, the integral Novikov conjecture in L-theory was proved by Ferry-Weinberger [FW] and Farrell-Jones [FJ]. On the other hand, among the family of discrete subgroups of Lie groups, the integral K-theoretic Novikov conjecture was only known for cocompact discrete subgroups [Ca] and arithmetic subgroups of linear algebraic groups [J] [Gol1]-[Gol3].

Received November 2, 2004

Acknowledgments: I would like to thank U.Bunke, M.Olbrich for helpful conversations on geometrically finite groups. This work was partially supported by NSF grants. 
For semisimple Lie groups $G$ of rank 1, in particular the (connected) group of isometries $S O(n, 1)$ of the real hyperbolic space $\mathbf{H}_{\mathbb{R}}^{n}$, another important class of discrete subgroups consists of geometrically finite discrete subgroups. They have played a crucial role in the theory of Kleinian groups [Ma], conformal analysis and low dimensional hyperbolic geometry [Ap1], and harmonic analysis and spectral theory [FHP1] [FHP2] [BO1] [BO2] [Co]. This class of discrete groups is rich, and there are many methods to construct them [Ma] [Ap1]. Arithmetic groups are geometrically finite. In fact, any co-finite discrete subgroup is geometrically finite. But there are many non-cofinite but geometrically finite discrete subgroups, for example, the convex co-compact Kleinian groups, and many groups constructed from the combination methods in $[\mathrm{Ma}]$ are not co-finite. We will show that the integral K-theoretic Novikov conjecture holds for these geometrically finite discrete subgroups of rank 1 semisimple Lie groups.

This note is simple and based on an observation in $[\mathrm{J}]$ on asymptotic dimension and some basic results on geometrically finite groups in [AX]. Probably the point of this note is to relate this important class of geometrically finite groups to problems around the Novikov conjectures. In fact, this connection occurred to the author during a lecture by U.Bunke at Oberwolfach in October, 2003, on spectral theory of such groups. Another motivation is that the list of groups which have both finite asymptotic dimension and finite classifying spaces is quite limited, and geometrically finite groups provide another natural class of groups which has both finiteness properties.

When $\Gamma$ is an arithmetic subgroup of a linear semisimple algebraic group $\mathbf{G}$, there is a well-known compactification $\overline{\Gamma \backslash X}^{B S}$ of the associated locally symmetric space $\Gamma \backslash X$ in [BS], called the Borel-Serre compactification, where $X=G / K$, $G=\mathbf{G}(\mathbb{R})$ and $K \subset G$ is a maximal compact subgroup of $G$. The compactification $\overline{\Gamma \backslash X}^{B S}$ has played an important role in analysis, geometry and topology of $\Gamma$. It turns out that when $\Gamma$ is geometrically finite, there is an analogous compactification of the associated locally symmetric space in [AX] (see Proposition 3.5 below), which is crucial for this note. Another purpose of this note is to explain this compactification and to make it better known.

The rest of this note is organized as follows. In $\S 2$, we recall the definition of geometrically finite groups $\Gamma$ and their basic properties. In $\S 3$, we recall a compactification of the associated locally symmetric space $\Gamma \backslash X$ in $[\mathrm{AX}]$ and use it to prove the Novikov conjecture. 


\section{Geometrically finite groups}

Let $G$ be a connected semisimple Lie group of rank $1, K \subset G$ a maximal compact subgroup. Then $X=G / K$ with any $G$-invariant Riemannian metric is a Riemannian symmetric space of noncompact type of rank 1 . It is known that $X$ is a hyperbolic space $\mathbf{H}_{K}^{n}$, where (1) $K$ is the field of real numbers $\mathbb{R}$ and $n \geq 2$, (2) $K$ is the field of complex numbers $\mathbb{C}$ and $n \geq 2$, (3) $K$ is the quaternion algebra and $n \geq 2$, and (4) $K$ is the algebra of octonions and $n=2$. (See [Mo, $\S 19]$ for more details). When $X$ is the real hyperbolic space $\mathbf{H}_{\mathbb{R}}^{n}$, we can take $G=S O(n, 1)$ and $K=S O(n)$.

Let $\Gamma \subset G$ be a discrete subgroup of $G$. If $\Gamma$ is finitely generated, then it is known by the so-called Selberg Lemma that $\Gamma$ admits a torsion free subgroup of finite index. In the following, we always assume that $\Gamma$ is torsion free, and hence that $\Gamma \backslash X$ is a manifold.

Let $X(\infty)$ be the set of equivalence classes of geodesics in $X$, the so-called sphere at infinity, and $X \cup X(\infty)$ the geodesic compactification. Then the isometric action of $G$ on $X$ extends continuously to $X \cup X(\infty)$. Since the rank of $X$ is equal to $1, X \cup X(\infty)$ is isomorphic to the maximal Satake compactification and hence is a real analytic manifold with boundary (see [BS] [BJ, §1.18]), and the extended action of $G$ on $X \cup X(\infty)$ is real analytic.

An element $g \in \Gamma$ is called parabolic if the extended action of $g$ on $X \cup X(\infty)$ has a unique fixed point in $X(\infty)$. A point $p \in X(\infty)$ is called a parabolic fixed point of $\Gamma$ if there exists a parabolic element of $\Gamma$ which fixes $p$. A subgroup of $\Gamma$ is called a parabolic subgroup if it consists of only parabolic elements and hence fixes a unique point in $X(\infty)$. Then it is known that for any parabolic fixed point $p$, the stabilizer $\Gamma_{p}=\{\gamma \in \Gamma \mid \gamma p=p\}$ consists of only parabolic elements (together with the identity element) and is a maximal parabolic subgroup of $\Gamma$.

Remark 2.1 In the above discussions, we follow the definition of parabolic subgroups in the theory of Kleinian groups, which is different from the notion in theories of Lie groups and linear algebraic groups. The latter will also be used in this note.

Let $\Lambda(\Gamma) \subset X(\infty)$ be the set of limit points of $\Gamma$, i.e., the set of accumulation points of any orbit $\Gamma \cdot x$ in $X \cup X(\infty)$, where $x \in X$. The complement $\Omega(\Gamma)=$ $X(\infty)-\Lambda(\Gamma)$ is called the domain of discontinuity, and $\Gamma$ acts properly on $\Omega(\Gamma)$.

When $X=\mathbf{H}_{\mathbb{R}}^{3}$, the real hyperbolic space of dimension $3, X(\infty)$ can be identified with the completed complex plane $S^{2}=\mathbb{C} \cup\{\infty\}$, and $\Gamma$ acts conformally on $S^{2}$. In this case, if $\Omega(\Gamma) \neq \emptyset, \Gamma$ is called a Kleinian group in complex analysis. 
For general $X, \Gamma$ is also called a Kleinian group if $\Omega(\Gamma) \neq \emptyset$. If $\Gamma$ is an arithmetic subgroup or more generally a co-finite subgroup, then $\Lambda(\Gamma)=X(\infty)$, and hence $\Gamma$ is not a Kleinian group.

Since $X \cup \Omega(\Gamma)$ is a real analytic manifold with boundary and $\Gamma$ acts real analytically, freely and properly on $X \cup \Omega(\Gamma)$, the quotient $\Gamma \backslash X \cup \Omega(\Gamma)$ is a real analytic manifold with boundary.

Definition 2.2 A discrete subgroup $\Gamma \subset G$ is called geometrically finite if $\Gamma \backslash X \cup$ $\Omega(\Gamma)$ has finitely many ends, each of which corresponds to a $\Gamma$-orbit of parabolic fixed points and hence to a $\Gamma$-conjugacy class of maximal parabolic subgroups of $\Gamma$, and each end is isomorphic to the standard end of a maximal parabolic subgroup of $\Gamma$.

More precisely, let $p \in X(\infty)$ be a parabolic fixed point of $\Gamma$ and $\Gamma_{p}$ its stabilizer. Then $\Gamma_{p} \backslash X \cup \Omega\left(\Gamma_{p}\right)$ has only one end, called the standard end associated with the parabolic subgroup $\Gamma_{p}$. Neighborhoods of the standard end will be described explicitly below and are called cusp regions.

Assume that $\Gamma$ is geometrically finite, and let $\Gamma_{p_{1}}, \cdots, \Gamma_{p_{m}}$ be a set of representatives of $\Gamma$-conjugacy classes of maximal parabolic subgroups of $\Gamma$, and $C_{p_{1}}, \cdots, C_{p_{m}}$ the cusp regions of $\Gamma_{p_{1}}, \cdots, \Gamma_{p_{m}}$ respectively. When they are sufficiently small, $C_{p_{1}}, \cdots, C_{p_{m}}$ can be identified with disjoint subsets of $\Gamma \backslash \cup \Omega\left(\Gamma_{p}\right)$, and the complement $\Gamma \backslash X \cup \Omega(\Gamma)-\cup_{i=1}^{m} C_{p_{i}}$ is compact.

By the reduction theory for cofinite discrete groups in a semisimple Lie group $G$ of rank 1 in [GR], when $\Gamma$ is such a cofinite discrete subgroup, $\Gamma \backslash X$ has finitely many ends, and each end is the product of $[0,+\infty)$ and a compact nilmanifold. Hence any cofinite discrete subgroup of a rank 1 semisimple Lie group $\Gamma$ is geometrically finite.

Another example is given by convex cocompact subgroups $\Gamma$. Briefly, let $C H(\Gamma)$ be the convex hull of $\Lambda(\Gamma)$ in $X \cup X(\infty)$. Then $\Gamma$ is called convex cocompact if the quotient $\Gamma \backslash C H(\Gamma) \cap X$ is compact, which is called the convex core of $\Gamma \backslash X \cup \Omega(\Gamma)$. Equivalently, $\Gamma$ is convex cocompact if $\Gamma$ is geometrically finite and does not contain any parabolic element, and hence $\Gamma \backslash X \cup \Omega(\Gamma)$ is a compact manifold with boundary. One simple example of convex cocompact subgroups is a Fuchsian group acting on $S^{2}$ which does not contain any parabolic element but considered as a discrete subgroup acting isometrically on $\mathbf{H}_{\mathbb{R}}^{3}$.

A basic result in [Bow2] is the following.

Proposition 2.3 If $\Gamma$ is geometrically finite, then $\Gamma$ is finitely generated. 
If $X=\mathbf{H}_{\mathbb{R}}^{n}$, it was proved further in [Bow1] that $\Gamma$ is finitely presented. For other $X$, it was proved in [AX, Corollary 6.6] that geometrically finite $\Gamma$ are also finitely presented, which follows from [AX, Theorem 6.5] recalled below in Proposition 3.5.

Remark 2.4 There are several equivalent definitions of geometrically finite groups in the theory of Kleinian group theory. For Fuchsian groups acting on $\mathbf{H}_{\mathbb{R}}^{2}$ and Kleinian groups acting on $\mathbf{H}_{\mathbb{R}}^{3}$, geometrically finite groups were first defined as discrete subgroups which admit covex (Dirichlet) fundamental domains bounded by finitely many totally geodesic hypersurfaces. It is known that in these two cases, finite generation of $\Gamma$ implies that $\Gamma$ is geometrically finite. This fact was used crucially to study the limit set $\Lambda(\Gamma)$. But this conclusion does not hold for $\mathbf{H}_{\mathbb{R}}^{n}$ in dimension $n \geq 4$. Because of this, for $\mathbf{H}_{\mathbb{R}}^{n}, n \geq 4$, convex fundamental domains are replaced by a union of finitely many polyhedrons each of which has finitely many sides. For other hyperbolic (i.e., rank-1 symmetric) spaces, there are no totally geodesic hypersurfaces, and geometrically finite groups are defined either in terms of the end structures of $\Gamma \backslash X \cup \Omega(\Gamma)$ as in Definition 2.2 above, or in terms of how points in the orbit $\Gamma \cdot x$ approach the points in the limit set $\Lambda(\Gamma)$, or in terms of compactness of the thick part of the convex core $\Gamma \backslash C H(\Gamma) \cap X$ or finite volume of any $\varepsilon$-neighborhood of the convex core. For detailed discussions of the history and equivalence of these different definitions, see [Bow1] [Bow2] [Ap1] [Ap2] [Ma].

Remark 2.5 There are several methods of constructing geometrically finite groups starting from simpler geometrically finite groups via the combination methods, which correspond to free products, amalgated products, and HNN-extensions [Ma] [Ap2]. Since free groups and Fuchsian groups are geometrically finite, many examples of geometrically finite groups can be constructed. For the real hyperbolic space $\mathbf{H}_{\mathbb{R}}^{n}$, suitable polyhedrons generate geometrically groups [Ma] [Ap1]. It is also conjectured (see [Bow1]) that any finitely generated discrete groups acting on $\mathbf{H}_{\mathbb{R}}^{n}$ are also algebraic limits of geometrically finite groups. Many important conjectures and problems about hyperbolic manifolds have been solved first for geometrically finite groups, and the study of geometrically finite groups has been crucial in low dimensional topology [Ap1] [Ap2] [Ma]. Therefore, the class of geometrically finite discrete groups is a very natural and important one.

\section{Compactification and the Novikov conjecture}

In this section, we prove the main result in this note. 
Theorem 3.1 Let $X$ be any noncompact symmetric space of rank 1. If $\Gamma$ is a torsion free geometrically finite group acting isometrically on $X$, then the integral K-theoretic Novikov conjecture holds for $\Gamma$.

We first recall an approach in $[\mathrm{Yu}][\mathrm{Ba}][\mathrm{CG}]$ to prove the Novikov conjecture. Then we recall an observation on asymptotic dimension in $[\mathrm{J}]$ and a suitable compactification of $\Gamma \backslash X$ in $[\mathrm{AX}]$ to show that the conditions in this approach are satisfied.

For any metric space $(M, d)$, Gromov introduced an asymptotic dimension $\operatorname{asdim}(M)$ in $[\mathrm{Gro}]$. The asymptotic dimension $\operatorname{asdim}(\Gamma)$ of a finitely generated group $\Gamma$ is defined to be the asymptotic dimension of the Cayley graph $\operatorname{Cay}(\Gamma, S)$ of $\Gamma$ associated to any finite symmetric set $S$ of generators (or equivalently the asymptotic dimension of $\Gamma$ with the associated word metric $d_{S}$ ), which can be shown to be independent of the choice of the set $S$ of the generators. A group $\Gamma$ is said to have a finite classifying space (or $K(\Gamma, 1))$ if there is a $K(\Gamma, 1)$-space homotopically equivalent to a finite $\mathrm{CW}$-complex.

Motivated by an important result in $[\mathrm{Yu}]$ on the Novikov conjecture in $C^{*}$ algebras, Bartels $[\mathrm{Ba}]$ and Carlsson-Goldfarb $[\mathrm{CG}]$ proved the following.

Proposition 3.2 If a finitely generated torsion free group $\Gamma$ has finite asymptotic dimension and a finite classifying space, then the integral Novikov conjecture in algebraic K-theory holds for $\Gamma$.

For a geometrically finite discrete subgroup $\Gamma$, the finiteness of the asymptotic dimension follows from an observation in [J, Proposition 2.3] stated in the next result (Proposition 3.3), and the finiteness of a classifying space follows from the existence of a suitable compactification of $\Gamma \backslash X \cup \Omega(\Gamma)$ in $[\mathrm{AX}]$.

Proposition 3.3 If $X$ is a proper metric space, in particular a complete Riemannian manifold, and a group $\Gamma$ acts properly on $X$, then $\operatorname{asdim}(\Gamma) \leq \operatorname{asdim}(X)$.

Proof. For completeness, we include the simple proof. Let $S$ be a finite symmetric set of generators of $\Gamma$, and $d_{S}$ the associated word metric on $\Gamma$ (or its Cayley graph $\operatorname{Cay}(\Gamma, S))$. Let $d$ be the distance function on $X$. The idea is to show that for any point $x_{0} \in X$, the map $\left(\Gamma, d_{S}\right) \rightarrow \Gamma \cdot x_{0} \subset X, g \mapsto g x_{0}$, is a coarse equivalence to the orbit $\Gamma \cdot x_{0}$.

First we note that there exists a constant $C>0$ such that for any $\gamma, \delta \in \Gamma$,

$$
d\left(\gamma x_{0}, \delta x_{0}\right) \leq C d_{S}(\gamma, \delta) .
$$


Write $\gamma^{-1} \delta=g_{1} \cdots g_{n}$, where $g_{1}, \cdots, g_{n} \in S$, and $n=d_{S}(\gamma, \delta)$. Then

$$
\begin{aligned}
d\left(\gamma x_{0}, \delta x_{0}\right) & =d\left(x_{0}, \gamma^{-1} \delta x_{0}\right)=d\left(x_{0}, g_{1} \cdots g_{n} x_{0}\right) \\
& \leq d\left(x_{0}, g_{1} x_{0}\right)+d\left(g_{1} x_{0}, g_{1} g_{2} x_{0}\right)+\cdots+d\left(g_{1} \cdots g_{n-1} x_{0}, g_{1} \cdots g_{n} x_{0}\right) \\
& =d\left(x_{0}, g_{1} x_{0}\right)+d\left(x_{0}, g_{2} x_{0}\right)+\cdots+d\left(x_{0}, g_{n} x_{0}\right) \\
& \leq C n=C d_{S}(\gamma, \delta),
\end{aligned}
$$

where $C=\max \left\{d\left(x_{0}, g x_{0}\right) \mid g \in S\right\}$.

On the other hand, since $(X, d)$ is a proper metric space, for any $r \geq 0$, the ball $B\left(x_{0}, r\right)=\left\{x \in X \mid d\left(x, x_{0}\right) \leq r\right\}$ is compact. By assumption, $\Gamma$ acts properly on $X$, and hence the set

$$
I_{r}=\left\{\gamma \in \Gamma \mid d\left(\gamma x_{0}, x_{0}\right) \leq r\right\}=\left\{\gamma \in \Gamma \mid \gamma\left\{x_{0}\right\} \cap B\left(x_{0}, r\right) \neq \emptyset\right\}
$$

is finite. Define

$$
f(r)=1+\max \left\{d_{S}(\gamma, e) \mid \gamma \in I_{r}\right\} .
$$

Clearly $f(r)$ is a finite positive increasing function. We claim that for any $\gamma, \delta \in \Gamma$,

$$
d_{S}(\gamma, \delta) \leq f\left(d\left(\gamma x_{0}, \delta x_{0}\right)\right) .
$$

In fact, let $r=d\left(\gamma x_{0}, \delta x_{0}\right)$. Then $d\left(\delta^{-1} \gamma x_{0}, x_{0}\right)=r$, and hence $\delta^{-1} \gamma \in I_{r}$. By definition, $d_{S}\left(\delta^{-1} \gamma, e\right) \leq f(r)$, which is equivalent to

$$
d_{S}(\gamma, \delta) \leq f\left(d\left(\gamma x_{0}, \delta x_{0}\right)\right) .
$$

Since the map $\Gamma \rightarrow \Gamma x_{0}$ is proper, this proves that the map $\left(\Gamma, d_{S}\right) \rightarrow\left(\Gamma x_{0}, d\right)$ is a coarse equivalence. Hence $\operatorname{asdim} \Gamma=\operatorname{asdim}\left(\Gamma x_{0}, d\right) \leq \operatorname{asdim} M$.

Corollary 3.4 For any symmetric space $X$ of noncompact type of rank 1, if $\Gamma$ acts properly on $X$, in particular if $\Gamma$ is geometrically finite, then $\operatorname{asdim}(\Gamma) \leq$ $\operatorname{dim} X$.

Proof. Since $X$ has strictly negative sectional curvature, by [Gro, Example 1. $\left.E_{1}^{\prime}\right], \operatorname{asdim}(X)=\operatorname{dim} X$. By Proposition 3.3, $\operatorname{asdim}(\Gamma) \leq \operatorname{asdim}(X)=\operatorname{dim}(X)$.

To prove Theorem 3.1, it remains to construct a finite classifying space of $\Gamma$. Since $\Gamma$ is torsion free and $X$ is contractible, $\Gamma \backslash X$ is a $K(\Gamma, 1)$-space.

If $\Gamma \backslash X$ is compact, then it is a compact (real analytic) manifold and hence admits a finite triangulation and is a finite classifying space of $\Gamma$. 
Assume that $\Gamma \backslash X$ is not compact. If we can construct a compactification $\overline{\Gamma \backslash X}$ of $\Gamma \backslash X$ which is a compact manifold with corners with interior equal to $\Gamma \backslash X$, then the inclusion $\Gamma \backslash X \rightarrow \overline{\Gamma \backslash X}$ is a homotopy equivalence. Since a compact manifold with corners admits a finite triangulation, the compactification $\overline{\Gamma \backslash X}$ is a finite classifying space of $\Gamma$. This will complete the proof of Theorem 3.1.

Proposition 3.5 Let $\Gamma$ be a torsion free geometrically finite group as above. Assume that $\Gamma \backslash X$ is noncompact. Then $\Gamma \backslash X$ admits a compactification $\overline{\Gamma \backslash X}$ which is a real analytic manifold with corners whose interior is equal to $\Gamma \backslash X$.

This result is proved in [AX, Theorem 6.5]. In fact, the result in $[\mathrm{AX}]$ deals with a more general class of quotients of pinched Hadamard manifolds having N-property, though symmetric spaces of rank 1 are the main examples of pinched Hadamard manifolds having N-property. It is stated in $[\mathrm{AX}]$ that the compactification $\overline{\Gamma \backslash X}$ is a compact manifold with boundary which is obtained from $\Gamma \backslash X \cup \Omega(\Gamma)$ by adding a virtually nilpotent manifold $\Gamma_{p} \backslash \mathcal{N}_{\Gamma_{p}}$ to the end corresponding to the maximal parabolic subgroup $\Gamma_{p}$. Actually, we need to add a manifold with boundary which is a fiber bundle over $\Gamma_{p} \backslash \mathcal{N}_{\Gamma_{p}}$ with the fiber being a compact manifold with boundary, and hence the resulting compactification is a manifold with corners instead of a manifold with boundary.

Remark 3.6 Presumably, by a manifold in $[\mathrm{AP}]$, it means a differential $\left(C^{\infty}\right)$ manifold. The reason for getting a real analytic manifold with corners is suggested by the Borel-Serre compactification of quotients of symmetric spaces by arithmetic groups, which is a real analytic manifold with corners (see [BS] [BJ, $\S 3.5, \S 3.9]$ ) and has played an important role in the study of cohomology of arithmetic groups. A natural question is to construct a similar compactification of $\Gamma \backslash X$ for geometrically finite $\Gamma$. When $\Gamma$ is co-finite, the compactification $\overline{\Gamma \backslash X}$ in the above proposition is the same as the Borel-Serre compactification, and hence $\overline{\Gamma \backslash X}$ is a natural generalization of the Borel-Serre compactification.

Remark 3.7 According to Proposition 3.2, a natural class of groups consists of those having both finite asymptotic dimension and finite classifying spaces. A lot of work has been done to understand groups with finite asymptotic dimension (See $[\mathrm{Dr}][\mathrm{Be}][\mathrm{BD} 1][\mathrm{BD} 2])$. On the other hand, less is understood which groups have finite classifying spaces. An important difference between these two finiteness properties is that the finiteness of asymptotic dimension is preserved after passing to a finitely generated subgroup, but the finiteness of the classifying space is not.

Remarks 3.8 When $X=\mathbf{H}_{\mathbb{R}}^{3}$, a different compactification of $\Gamma \backslash X$ or $\Gamma \backslash X \cup \Omega(\Gamma)$ was constructed in [FHP1, p. 298] by adding a circle to each end of $\Gamma \backslash X \cup \Omega(\Gamma)$, 
and is useful for spectral theory of $\Gamma \backslash X$. This compactification is a quotient of the compactification $\overline{\Gamma \backslash X}$ in the above proposition in the sense that there is a continuous, surjective map from $\overline{\Gamma \backslash X}$ to it extending the identity map on $\Gamma \backslash X$.

Other useful compactifications of $\Gamma \backslash X$ are also obtained by adding suitable curves to each end of $\Gamma \backslash X \cup \Omega(\Gamma)$ so that the resulting compactifications are smooth manifolds [Ma, p. 128-130].

Remark 3.9 For a compactification $\bar{M}$ of a noncompact manifold $M$, the inclusion $M \subset \bar{M}$ is not necessarily a homotopy equivalence if the interior of $\bar{M}$ strictly contains $M$. For example, $M=\mathbb{R}$ can be compactified to $\mathbb{R} \cup\{\infty\} \cong S^{1}$.

Remark 3.10 According to [Ap2, Theorem 7.59, p. 302], any three dimensional geometrically tame hyperbolic manifold $M$ admits a compactification which is a topological manifold with corners whose interior is equal to $M$. Since geometrically finite groups are geometrically tame, this also implies that Theorem 3.1 holds for this more general class of Kleinian groups acting on $\mathbf{H}_{\mathbb{R}}^{3}$.

The rest of this note is to prove Proposition 3.5 and hence Theorem 3.1. For completeness, we recall the proof from $[\mathrm{AX}]$ with more details and comment how the corner structure arises and how the real analytic structure of $\Gamma \backslash X$ can be extended to the compactification $\overline{\Gamma \backslash X}$.

If $\Gamma$ does not contain any parabolic element, i.e., $\Gamma$ is convex cocompact, then $\Gamma \backslash X \cup \Omega(\Gamma)$ is a compact real analytic manifold with boundary, a desired compactification of $\Gamma \backslash X$. In fact, we note that since $\Gamma$ is torsion free, $\Gamma$ acts freely on $X$. It also acts freely on $\Omega(\Gamma)$ since it does not contain any parabolic element. Furthermore, as pointed out at the beginning of $\S 2, X \cup \Omega(\Gamma)$ is a real analytic manifold with boundary, and the $\Gamma$-action on $X$ extends to a real analytic action on $X \cup \Omega(\Gamma)$.

Otherwise, $\Gamma$ contains parabolic elements, and we need to construct a compactification of $\Gamma \backslash X \cup \Omega(\Gamma)$ and hence a compactification of $\Gamma \backslash X$. By the definition of geometrical finiteness, the ends of $\Gamma \backslash X \cup \Omega(\Gamma)$ are given by the standard end of $\Gamma_{p} \backslash X \cup \Omega\left(\Gamma_{p_{i}}\right)$, where $\Gamma_{p_{i}}, i=1, \cdots, m$, are representatives of $\Gamma$-conjugacy classes of maximal parabolic subgroups of $\Gamma$. Therefore, it suffices to construct a compactification of $\Gamma_{p_{i}} \backslash X \cup \Omega\left(\Gamma_{p_{i}}\right)$ for each $\Gamma_{p_{i}}$. For simplicity, we denote $p_{i}$ by $p$.

Since the case $X=\mathbf{H}_{\mathbb{R}}^{n}$ is the simplest and most important, we discuss it first. In the upper half space model,

$$
\mathbf{H}_{\mathbb{R}}^{n}=\left\{\left(x_{1}, \cdots, x_{n}\right) \mid x_{1}, \cdots, x_{n-1} \in \mathbb{R}, x_{n}>0\right\} .
$$


Assume that the parabolic fixed point $p$ is equal to $\infty$. Then the horospheres of $p$ are given by $N\left(x_{n, 0}\right)=\left\{\left(x_{1}, \cdots, x_{n}\right) \mid x_{1}, \cdots, x_{n-1} \in \mathbb{R}, x_{n}=x_{n, 0}\right\}$, where $x_{n, 0}$ is any fixed positive number, and the parabolic subgroup $\Gamma_{p}$, also denoted by $\Gamma_{\infty}$, preserves each horosphere. Identify $N\left(x_{n, 0}\right)$ with $\mathbb{R}^{n-1}$. Then $\Gamma_{\infty}$ acts isometrically on $\mathbb{R}^{n-1}$ with respect to the standard Euclidean metric.

By the Bieberbach Theorem [Ap1, Theorem 3.4] [Bow1, Theorem 2.2.5, Proposition 2.2.6], there is a subspace $V$ of $\mathbb{R}^{n-1}$ which is invariant under $\Gamma_{\infty}$ and the quotient $\Gamma_{\infty} \backslash V$ is compact. Let $d$ be the distance function on $\mathbb{R}^{n}$ with respect to the standard Euclidean metric. Identify

$$
\mathbf{H}_{\mathbb{R}}^{n} \cong \mathbb{R}^{n-1} \times \mathbb{R}_{>0}, \quad \mathbf{H}_{\mathbb{R}}^{n} \cup\left(\mathbf{H}_{\mathbb{R}}^{n}(\infty)-\{\infty\}\right) \cong \mathbb{R}^{n-1} \times \mathbb{R}_{\geq 0}
$$

and

$$
\mathbb{R}^{n-1} \cong \mathbb{R}^{n-1} \times\{0\} \subset \mathbb{R}^{n-1} \times \mathbb{R}_{\geq 0}
$$

For any $r>0$, define

$$
C_{\infty}(V, r)=\left\{x=\left(x_{1}, \cdots, x_{n}\right) \in \mathbf{H}_{\mathbb{R}}^{n} \cup\left(\mathbf{H}_{\mathbb{R}}^{n}(\infty)-\{\infty\}\right) \mid d(x, V)>r\right\} .
$$

Then $C_{\infty}(V, r)$ is invariant under $\Gamma_{\infty}$, and the quotient $\Gamma_{\infty} \backslash C_{\infty}(V, r)$ is a standard cusp region of the unique end of $\Gamma_{\infty} \backslash X \cup \Omega\left(\Gamma_{\infty}\right)$.

If $\operatorname{dim} V=n-1$, i.e., $\Gamma_{\infty}$ is a parabolic subgroup of full rank, then $C(V, r)$ is a half-space, and $\Gamma_{\infty} \backslash C_{\infty}(V, r)$ is the product of a (virtual) torus $\Gamma \backslash \mathbb{R}^{n-1}$ and $(r,+\infty)$. Otherwise, $\Gamma_{\infty} \backslash C_{\infty}(V, r)$ is virtually the product of a torus and a vector space.

For any $R>r$, define

$$
S_{\infty}(V, R)=\left\{x \in C_{\infty}(V, r) \mid d(x, V)=R\right\} .
$$

Clearly, $S_{\infty}(V, R)$ is a real analytic manifold with boundary, and for different values of $R, S_{\infty}(V, R)$ are real analytically diffeomorphic to each other. Fix any $R_{0}>r$. Then $C_{\infty}(V, r)$ is real analytically diffeomorphic to $S_{\infty}\left(V, R_{0}\right) \times(r,+\infty)$. Define

$$
\overline{C_{\infty}(V, r)} \cong S_{\infty}\left(V, R_{0}\right) \times(r,+\infty] .
$$

Since the $\Gamma_{p}$-action on $C_{\infty}(V, r)$ preserves each of both factors $S_{\infty}(V, R)$ and $(0,+\infty)$ in the product, it extends to a real analytic free action on $\overline{C_{\infty}(V, r)}$. The quotient $\Gamma_{\infty} \backslash \overline{C_{\infty}(V, r)}$ is a real analytic manifold with corners and can be used to compactify $\Gamma_{\infty} \backslash X \cup \Omega\left(\Gamma_{\infty}\right)$ into a real analytic manifold with corners. Clearly the interior of $\overline{\Gamma \backslash X}$ is equal to $\Gamma \backslash X$ and hence the inclusion $\Gamma \backslash X \subset \overline{\Gamma \backslash X}$ is a homotopy equivalence. This proves Proposition 3.5 for the case of $X=\mathbf{H}_{\mathbb{R}}^{n}$. 
In general, for a noncompact symmetric space $X=G / K$ of rank 1 and any parabolic fixed point $p \in X(\infty)$, the stabilizer $G_{p}$ of $p$ is a parabolic subgroup of the Lie group $G$. Let $N$ be the unipotent radical of $G_{p}$. Then $N$ acts simply transitively on each of the horospheres of $p$. Identifying these horospheres of $p$ with $N$, we obtain a horospherical decomposition $X=N \times \mathbb{R}_{>0}$, a generalization of the upper space model of $\mathbf{H}_{\mathbb{R}}^{n}$.

Since $\Gamma_{p}$ is not necessarily contained in $N$, we need a group theoretical interpretation of the horospherical decomposition. Let $\mathfrak{g}$ be the Lie algebra of $G$, $\mathfrak{k}$ the Lie algebra of $K$. Let $\mathfrak{g}=\mathfrak{k} \oplus \mathfrak{p}$ be the Cartan decomposition associated with $K$. Let $\mathfrak{a} \subset \mathfrak{p}$ be a maximal abelian subalgebra contained in the Lie algebra $\mathfrak{p}$ of $G_{p}$. By the rank 1 assumption on $X, \operatorname{dim} \mathfrak{a}=1$. Then $A=\exp \mathfrak{a}$ is the split component of $G_{p}$. Let $M$ be the centralizer of $A$ in $K$. Then the parabolic subgroup $G_{p}$ admits the Langlands decomposition $G_{p}=N A M$. Identify $\mathfrak{a} \cong \mathbb{R}$ and $A \cong \mathbb{R}_{>0}$. Since $G_{p}$ acts transitively on $X$, the Langlands decomposition of $G_{p}$ gives the horospherical decomposition of $X$ :

$$
X=N A x_{0} \cong N \times A \cong N \times \mathbb{R}_{>0}=N \times(0,+\infty),
$$

where $x_{0}=K$ is a basepoint.

In the following, we identify $N$ with the orbit $N x_{0}$ in $X$ and endow it with the invariant Riemannian metric induced from $X$. (The distance function induced from this invariant Riemannian metric on $N$ is written down explicitly in [AX, $\S 2])$. Then $N M$ acts isometrically on $N$. Since the parabolic subgroup $\Gamma_{p}$ of $\Gamma$ is contained in $N M$, it also acts isometrically on $N$.

Since the rank of $X$ is equal to $1, X(\infty)-\{p\}$ can be naturally identified with $N$. Combined with the horospherical decomposition, we obtain the identification

$$
X \cup(X(\infty)-\{p\}) \cong N \times[0,+\infty),
$$

a real analytic diffeomorphism. The action of $N M$ on $X \cup X(\infty)$ preserves $X \cup(X(\infty)-\{\infty\})$ and the product structure in the above equation. Since $M$ commutes with $A$, it leaves the factor in $[0,+\infty)$ invariant.

Take the standard distance function on $[0,+\infty)$. Then its product with the distance of $N$ induced from the invariant Riemannian metric defines a distance function, denoted by $d$, on $X \cup(X(\infty)-\{p\})$ which is invariant under $N M$ and hence $\Gamma_{p}$.

By a generalization of the Bieberbach Theorem in [AX, Theorem 4.1], there exists a subgroup $N^{\prime}$ of $N$ (or rather an orbit of $N^{\prime}$ in $N$ ) which is invariant under $\Gamma_{p}$ and the quotient $\Gamma_{p} \backslash N^{\prime}$ is compact. 
Identify $N$ with the subset $N \times\{0\}$ in $N \times[0,+\infty)$. Then $N^{\prime}$ is also identified with a subset of $N \times[0,+\infty)$. For any $r>0$, define

$$
C_{p}\left(N^{\prime}, r\right)=\left\{x \in X \cup(X(\infty)-\{p\}) \mid d\left(x, N^{\prime}\right)>r\right\} .
$$

Since $N^{\prime}$ and $d$ are invariant under $\Gamma_{p}, C_{p}\left(N^{\prime}, r\right)$ is invariant under $\Gamma_{p}$. The quotient $\Gamma_{p} \backslash C_{p}\left(N^{\prime}, r\right)$ is a neighborhood of the unique end of $\Gamma_{p} \backslash X \cup \Omega\left(\Gamma_{p}\right)$.

For any $R>r$, let

$$
S_{p}\left(N^{\prime}, R\right)=\left\{x \in C_{p}\left(N^{\prime}, r\right) \mid d\left(x, N^{\prime}\right)=R\right\} .
$$

Since the distance $d$ is the product of the invariant distance function of $N$ and the standard Euclidean one on $[0,+\infty), d(x, y)$ is analytic in $x, y$ with non-zero gradient when $x \neq y$, and $S_{p}\left(N^{\prime}, R\right)$ is a real analytic manifold with boundary. Therefore, for different values of $R, S_{p}\left(N^{\prime}, R\right)$ are real analytically diffeomorphic to each other. This implies that for any fixed $R_{0}>r$,

$$
C_{p}\left(N^{\prime}, r\right) \cong S\left(N^{\prime}, R_{0}\right) \times(r,+\infty)
$$

as real analytic manifolds. Adding $S\left(N^{\prime}, R_{0}\right)$ at infinity, we obtain a real analytic manifold with corners

$$
\overline{C_{p}\left(N^{\prime}, r\right)} \cong S\left(N^{\prime}, R_{0}\right) \times(r,+\infty] .
$$

As in the case of $X=\mathbf{H}_{\mathbb{R}}^{n}$, the free real analytic action of $\Gamma_{p}$ on $C_{p}\left(N^{\prime}, r\right)$ extends to a free real analytic action on $\overline{C_{p}\left(N^{\prime}, r\right)}$. The quotient $\Gamma_{p} \backslash \overline{C_{p}\left(N^{\prime}, r\right)}$ is a real analytic manifold with corners, and can be used to compactify $\Gamma_{p} \backslash X \cup \Omega\left(\Gamma_{p}\right)$ into a real analytic manifold with corners. The interior of $\overline{\Gamma \backslash X}$ is clearly equal to $\Gamma \backslash X$. This completes the proof of Proposition 3.5 and hence Theorem 3.1.

\section{References}

[Ap1] B.Apanasov, Conformal geometry of discrete groups and manifolds, Walter de Gruyter, 2000.

[Ap2] B.Apanasov, Discrete groups in space and uniformization problems, Kluwer Academic Publishers, 1991.

[AX] B.Apanasov, X.Xie, Discrete actions on nilpotent Lie groups and negatively curved spaces, Diff. Geom and its Appl. 20 (2004) 11-29.

[Ba] A.Bartels, Squeezing and higher algebraic K-theory, K-Theory 28 (2003) $19-37$. 
[BFJR] A.Bartels, T.Farrell, L.Jones, H.Reich, On the isomorphism conjecture in algebraic K-theory, to appear in Topology, 2003.

[BE] G.Bell, Asymptotic properties of groups acting on complexes, preprint, 2002 .

[BD1] G.Bell, A.Dranishnikov, On asymptotic dimension of groups, Algebr. and Geom. Topol. 1 (2001), 57-71.

[BD2] G.Bell, A.Dranishnikov, On asymptotic dimension of groups, Algebr. and Geom. Topol. 1 (2001), 57-71.

[BJ] A.Borel, L.Ji, Compactifications of symmetric spaces and locally symmetric spaces, a volume in the series Mathematics: Theory and applications by Birkhäuser, to appear in 2005.

[BS] A.Borel, J.P.Serre, Corners and arithmetic groups, Comment. Math. Helv. 48 (1973), 436-491.

[Bow1] B.Bowditch, Geometrical finiteness for hyperbolic groups, J. Funct. Anal. 113 (1993) 245-317.

[Bow2] B.Bowditch, Geometrical finiteness with variable negative curvature, Duke Math. J. 77 (1995) 229-274.

[BO1] U.Bunke, M.Olbrich, Scattering theory for geometrically finite groups, preprint, 1999.

[BO2] U.Bunke, M.Olbrich, The spectrum of Kleinian manifolds, J. Funct. Anal. 172 (2000) 76-164.

[Ca] G.Carlsson, Bounded $K$-theory and the assembly map in algebraic $K$ theory, in Novikov conjectures, index theory and rigidity, vol. 2 (S.C.Ferry, A.Ranicki, J.Rosenberg, eds.), Cambridge U. Press, 1995, 5-127.

[CG1] G.Carlsson, B.Goldfarb, The integral K-theoretic Novikov conjecture for groups with finite asymptotic dimension, Invent. Math. 157 (2004) 405418.

[CG2] G.Carlsson, B.Goldfarb, On homological coherence of discrete groups, to appear in Journal of Algebra, http://math.albany.edu:8000/ goldfarb/.

[Co] K.Corlette, Hausdorff dimensions of limit sets. I, Invent. Math. 102 (1990) 521-541. 
[Dr] A.Dranishnikov, Dimension theory: local and global, preprint, Univ of Florida, http://www.math.ufl.edu/ dranish.

[FH] F.Farrell, W.Hsiang, On Novikov's conjecture for nonpositively curved manifolds, Ann. of Math. 113 (1981) 199-209.

[FJ1] F.Farrell, L.Jones, Isomorphism conjectures in algebraic K-theory, J.A.M.S 6 (1993) 249-297.

[FJ2] F.Farrell, L.Jones, Rigidity for aspherical manifolds with $\pi_{1} \subset G L_{m}(\mathbb{R})$, Asian J. Math. 2 (1998) 215-262.

[FRR] S.Ferry, A.Ranicki, J.Rosenberg, Novikov conjectures, index theorems and rigidity, vol. 1 \& 2, Cambridge University Press, 1995.

[FW] S.Ferry, S.Weinberger, Curvature, tangentiality, and controlled topology, Invent. Math. 105 (1991) 401-414.

[FHP1] R.Frose, P.Hislop, P.Perry, The Laplace operator on hyperbolic three manifolds with cusps of non-maximal rank, Invent. Math. 106 (1991) 295-333.

[FHP2] R.Frose, P.Hislop, P.Perry, A Mourre estimate and related bounds for hyperbolic manifolds with cusps of nonmaximal rank, J. Funct. Anal. 98 (1991) 292-310.

[GR] H.Garland, M.S.Raghunathan, Fundamental domains for lattices in ( $R$ Jank 1 semisimple Lie groups, Ann. of Math. 92(1970) 279-326.

[Gol1] B.Goldfarb, Novikov conjectures for arithmetic groups with large actions at infinity, K-theory, 11 (1997), 319-372.

[Gol2] B.Goldfarb, The large scale topology and algebraic K-theory of arithmetic groups, Topology Appl. 140 (2004) 267-294.

[Gol3] B.Goldfarb, Strong Novikov conjecture for lattices in $S L_{3}$, to appear in Proc. of 2003 Wasatch Topology Conf.

[Gro] M.Gromov, Asymptotic invariants of infinite groups, in Geometric group theory, ed. by A.Niblo and M.Roller, Cambridge University Press.

$[\mathrm{J}] \quad$ L.Ji, Asymptotic dimension and the integral K-theoretic Novikov conjecture for arithmetic groups, preprint.

[Ka] G.Kasparov, Equivariant KK-theory and the Novikov conjecture, Invent. Math. 91 (1988) 147-201. 
[Ma] B.Maskit, Kleinian groups, Springer-Verlag, 1998.

[Mo] G.Mostow, Strong rigidity of locally symmetric spaces, Princeton University Press, 1973.

[Ra] A.Ranicki, Algebraic L-theory and topological manifolds, Cambridge University Press, 1992

[Yu] G.Yu, The Novikov conjecture for groups with finite asymptotic dimension, Ann. of Math. 147 (1998), 325-355.

Department of Mathematics University of Michigan Ann Arbor, MI 48109.

E-mail address: lji@umich.edu 